\title{
A role for Follistatin-like protein 1 (FSTL1) in colorectal cancer
}

\author{
Ioannis A. Voutsadakis ${ }^{1,2}$ \\ ${ }^{1}$ Algoma District Cancer Program, Sault Area Hospital, Sault Ste. Marie, Ontario, Canada; ${ }^{2}$ Section of Internal Medicine, Division of Clinical \\ Sciences, Northern Ontario School of Medicine, Sudbury, Ontario, Canada \\ Correspondence to: Ioannis A. Voutsadakis, MD, PhD. Algoma District Cancer Program, Sault Area Hospital, 750 Great Northern Road, Sault Ste \\ Marie, ON P6B 0A8, Canada. Email: ivoutsadakis@yahoo.com or ivoutsadakis@nosm.ca. \\ Provenance and Peer Review: This article was commissioned by the Editorial Office, Annals of Translational Medicine. The article did not undergo \\ external peer review. \\ Comment on: Zhao Y, Ou Q, Deng Y, et al. Determination of follistatin-like protein 1 expression in colorectal cancer and its association with clinical \\ outcomes. Ann Transl Med 2019;7:606.
}

Submitted Dec 24, 2019. Accepted for publication Jan 09, 2020.

doi: 10.21037/atm.2020.01.112

View this article at: http://dx.doi.org/10.21037/atm.2020.01.112

Follistatin-like protein 1 [FSTL1, alternative names: TGF $\beta$ induced clone 36 (TSC36) or Follistatin-Related Protein (FRP)] is a glycosylated 308 amino-acid secreted protein expressed in various human tissues (1). It plays various roles in vertebrate development including the dorso-ventral axis establishment and skeletal, lung and ureter development (2). In mice, disruption of the $f s t l 1$ gene results in neonatal mortality hours after birth with respiratory distress (3). In human adult tissues FSTL1 mRNA is most abundantly expressed in adipose tissue, urinary bladder, prostate and endometrium (4). FSTL1 belongs to the SPARC (Secreted Protein Acid and Rich in Cysteine) family of proteins together with SPARC, FSTL4, FSTL5, SMOC1, SMOC2, hevin and testican-1, -2 and -3 . Similar to other members of the family, FSTL1 possesses a follistatin domain and an extracellular calcium-binding (EC) domain. In human FSTL1 these domains occupy a position from amino acids 31 to 98 and from 113 to 232 respectively (3). More amino-terminal (amino acids 1-20), FSTL1 possesses a signal domain required for secretion of the protein. Carboxyterminal to EC domain, from amino acids 232 to 271 of the protein, a von Willebrand C type domain is present, which functions in protein-protein interactions.

Besides its role in development, FSTL1 is involved in diverse pathologies that include cardiovascular and lung diseases, autoimmune diseases and cancer (1). The function of FSTL1 is mediated by interaction with proteins of the cell surface. Main interactors with FSTL1 are receptors of the transforming growth factor beta (TGF $\beta$ )/bone morphogenic protein (BMP) family and disconnected (disco)interacting protein 2 homolog A (DIP2A) protein (5). In the immune system FSTL1 is also engaged by CD14 and toll-like receptor 4 (TLR4), the latter is also expressed in colorectal cancer cells. Through these receptor interactions FSTL1 has pleotropic effects in cells that express the receptors in their surface. In cancer in particular, FSTL1 may influence cancer cells both through direct interaction with cells that express TGF $\beta / B M P$ family receptors and DIP2A protein and indirectly through effects on the immune system. Binding of FSTL1 with BMP receptors takes place in co-operation with ligands BMP2 and BMP4 and lead to inhibition of signal transduction from the receptor (3). In contrast, interactions with DIP2A and CD14/TLR4 activate down-stream signaling. In cancer, FSTL1 has been assigned diverse roles in progression and metastasis and is reported both to promote and impede carcinogenic processes in different cancer cells (6-9).

Since FSTL1 is a secreted protein and acts from outside a cell by interacting with proteins in the cell surface, its provenance could be from the receiver cell itself (autocrine action) or neighboring cells (paracrine action). The effect would depend on the expression of receptors interacting with FSTL1 in a cell's surface and on the abundance of the glycoprotein in a cell's micro-environment. FSTL1 is expressed in both fibroblast cell lines established from colon cancer patients and in colonic cancer cell lines (10). LoVo colon cancer cells injected in nude mice together with established fibroblast cell lines produced smaller 
tumors than LoVo cells injected alone, suggesting that factors in fibroblasts or secreted by fibroblasts inhibit cancer cells. Knock-down of FSTL1 with siRNA in cocultures of colon cancer cells with fibroblasts accelerated cancer cell proliferation (10). A study of colorectal cancer stroma identified FSTL1 as significantly increased in whole cell extracts and supernatants of colon cancer associated fibroblasts compared to normal fibroblasts (11). A reciprocal effect whence FSTL1 from cancer cells suppress immune cells in the metastatic tumor microenvironment has also been described (12). As a result, FSTL1 may have a role both in metastasis initiation, through TGF $\beta$ signaling which promotes induction of Epithelial to Mesenchymal Transition, and in metastasis establishment in remote sites $(13,14)$.

Zhao et al. report on the expression of FSTL1 in patients with colorectal cancer (15). They investigated levels of FSTL1 mRNA and protein expression. Expression of FSTL1 mRNA as determined by both transcriptome array and RT-qPCR was higher in colon cancer tissues of patients of various stages of colorectal cancer compared with paired non-tumor tissues. Moreover, circulating FSTL1 levels were significantly higher in the serum of colorectal cancer patients compared to healthy controls. Mean FSTL1 levels in colorectal cancer patients were $1.91 \mathrm{ng} / \mathrm{mL}$ (SD: 1.4) and those in the serum of healthy controls were $1.1 \mathrm{ng} / \mathrm{mL}$ (SD: 1.25). At the protein level which was examined by tissue microarrays, cancer cells of stage II to IV colorectal carcinoma patients expressed FSTL1 at higher levels than the adjacent normal colonic epithelium. In contrast, tumor stroma was shown to express FSTL1 at lower mean levels than the corresponding stroma of adjacent normal tissue. An overall survival (OS) analysis showed that patients with tumors with higher FSTL1 levels (above a cut-off of 6.5) had worse OS compared with patients whose tumors had lower FSTL1 expression levels (below the cut-off of 6.5) (15). In contrast, an additional analysis that stratified patients according to the tumor stroma expression of FSTL1 showed that patients whose tumors had a higher stromal expression of FSTL1 (above a cut-off of 3.17) had better OS than patients with lower expression of FSTL1 in the tumor stroma (below the cut-off of 3.17). The two analyses suggested that the favorable effect of FSTL1 for OS in tumor stroma may be more pronounced than the deleterious effect of higher levels of the protein when expressed in tumor cells. Thus, FSTL1 is elevated in colorectal cancer patients and its location is important for its biologic effects and prognostic repercussions. It should be noted that the cut-offs for FSTL1 proposed in the study are arbitrary, derived from optimization of differences between high and low expression categories and it is unknown if they carry a biologic significance. In addition, the study does not specify a minimum distance of normal tissues sampled from cancerous tissues (15). Survival data imply an association of FSTL1 with adverse prognosis in colorectal cancer with the additional caveat that the location of the protein within the tumor micro-environment may be critical. Overall these data are interpreted to signify an adverse effect of FSTL1 in colorectal cancer prognosis possibly derived from signaling through the BMP pathway. In cancer stroma FSTL1 may have tumor-suppressive effects through influence on tumor microenvironment, including immune cell effects. Given the diverse effects of FSTL1 it is plausible that its availability and abundance in various locations in the extracellular compartment will eventually define its net effect on a specific cancer.

How could all data on FSTL1 role in colorectal carcinogenesis be integrated to produce a unifying model that may help in prognostic models and therapies development? The TGF $\beta /$ BMP pathway is one of the most commonly affected pathways in colorectal cancer (16). TGF $\beta$ signaling switches from tumor suppressor in precancerous epithelium to tumor promoting in established cancers. The TGF $\beta$ branch tends to promote proliferation, depending on in-puts from additional pathways such as K-Ras, while the BMP branch tends to have tumor controlling influence (17). Several components of the TGF $\beta / B M P$ pathway may be mutated in colorectal cancer. For example, mutations of SMAD4, which serves as a common partner in both arms, lead to deregulation of the pro-carcinogenic activities of the TGF $\beta$ branch and regulatory activities of BMP branch (18). Inhibition of the BMP branch by interactions of FSTL1 with BMP receptors may accentuate the influence of pathway mutations and further tip the balance towards pro-carcinogenic actions. Alternatively, in cancers with no mutations activating TGF $\beta$ signaling, FSTL1 actions may be the primary cause of an imbalanced TGF $\beta / B M P$ pro-carcinogenic output. Increased TGF $\beta$ signaling is associated with proliferation, invasion, metastasis and chemoresistance in colorectal cancer (18). However, due to additional input of signals to the TGF $\beta / B M P$ pathway from other pathways such as $\mathrm{K}$-Ras, which may be aberrantly activated in a sub-set of colorectal cancers, the net effect of inhibitory engagement of BMP receptors by FSTL1 could be paradoxically deleterious for the cancer cell and thus explain the proliferation block observed in some studies (10). 
The expression of TLR4 (CD284), another FSTL1 interacting protein, is low in normal colonic epithelium but becomes up-regulated in inflammatory disorders such as inflammatory bowel disease (19). TLR4 engagement in colorectal cancer cells has direct effects in enhancing proliferation and inhibiting apoptosis (20). In addition, activation of TLR4 in intestinal mesenchymal cells and cancer associated fibroblasts promotes intestinal carcinogenesis in an APC-mutant mouse model (21). Deletion of the gene for TLR4 or the downstream signaling protein $\mathrm{MyD} 88$ suppresses tumor formation in this model (21).

In contrast to the direct effect of FSTL1 in colorectal cancer cells, in stroma, FSTL1 may influence colorectal cancer progression through interactions with immune cells by the engagement of CD14 and TLR4. Both these proteins have bacterial lipopolysaccharide as ligands, functioning as pattern recognition receptors in the sensing of microbial invasion. Cells that express CD14 include those of the monocytic/ macrophage lineage. In melanoma patients, the monocytic subset of myeloid-derived suppressor cells (moMDSCs) a subset of MDSCs displaying the phenotype CD14 $/$ HLA-DR ${ }^{\text {low }}$ negative is higher than in normal control patients without cancer and may play a role in immune suppression observed in cancer patients (22). In the tumor micro-environment moMDSCs could contribute to suppression of incoming tumor infiltrating effector T cells. FSTL1 may further promote moMDSCs action by triggering CD14.

FSTL1 has deleterious effects in anti-tumoral immunity through engagement of the DIP2A receptor in cancer cells which then signal to activate immune suppressing cells in the stroma (23). Epigenetic signaling of DIP2A leads to H3 histone deacetylation at lysine 9 (H3K9) through complex formation and activation of deacetylase HDAC2 (24). FSTL1 prevents the formation of the DIP2A-HDAC2 complex and leads to acetylation of $\mathrm{H} 3 \mathrm{~K} 9$ promoting expression of methyltransferase MGMT in glioblastoma cells and temozolamide resistance. Whether this resistance mechanism operates in other cancers and in colorectal cancer in particular remains to be confirmed. Epigenetic modulation may have much broader effects beyond those seen in the expression of MGMT and could prove to be a major player in the effects of FSTL1. Interestingly, another DIP2 homologue, DIP2C is also involved in epigenetic regulation of cancer cells and its loss results in hypomethylation of several hundreds of DNA sites (25). Genes whose expression is affected include cell cycle inhibitor CDKN2A, EMT master regulator ZEB1 and hyaluronic acid receptor and stem cell marker CD44.

FSTL1 role in colorectal cancer is complex and remains incompletely understood. Complexities in the tumor microenvironment with different cells responding to FSTL1 in variable ways certainly contribute to conflicting results between studies. Part of the ambiguities in the role of FSTL1 in cancer results from the fact that several processes in which it is involved are regulated at multiple layers and the same regulator may have contrasting results depending on the specific microenvironment. This is true for TGF $\beta$ signaling as alluded above, for cancer immunity where immune effectors and suppressors are at play, for EMT where the optimal state in cancer is defined by higher plasticity when cells can easily switch over from epithelial to mesenchymal state and back and for epigenetic changes where expression of multiple genes can be modulated at the same time from one epigenetic change, such as H3K9 acetylation. Studies including the one by Zhao et al. (15) approaching the role of FSTL1 from different angles will help further clarify FSTL1 influences in colorectal carcinogenesis. The final goal is to introduce FSTL1 in the clinic as a prognostic marker and possibly as a target for therapy. In this respect, a preclinical study of monoclonal antibodies blocking FSTL1 has shown a tumor suppressing effect in in vivo syngeneic and xenograft mouse models and could pave the way for further development, guided by predictive markers (23).

\section{Acknowledgments}

Funding: None.

\section{Footnote}

Conflicts of Interest: The author has no conflicts of interest to declare.

Ethical Statement: The author is accountable for all aspects of the work in ensuring that questions related to the accuracy or integrity of any part of the work are appropriately investigated and resolved.

Open Access Statement: This is an Open Access article distributed in accordance with the Creative Commons Attribution-NonCommercial-NoDerivs 4.0 International License (CC BY-NC-ND 4.0), which permits the noncommercial replication and distribution of the article with the strict proviso that no changes or edits are made and the original work is properly cited (including links to both the 
formal publication through the relevant DOI and the license). See: https://creativecommons.org/licenses/by-nc-nd/4.0/.

\section{References}

1. Mattiotti A, Prakash S, Barnett P, et al. Follistatin-like 1 in development and human diseases. Cell Mol Life Sci 2018;75:2339-54.

2. Sylva M, Li VSX, Buffing AAA, et al. The BMP Antagonist Follistatin-Like 1 Is Required for Skeletal and Lung Organogenesis. PLoS One 2011;6:e22616.

3. Sylva M, Moorman AF, van den Hoff MJ. Follistatin-like 1 in vertebrate development. Birth Defects Res C Embryo Today 2013;99:61-9.

4. Fagerberg L, Hallström BM, Oksvold P, et al. Analysis of the human tissue-specific expression by genomewide integration of transcriptomics and antibody-based proteomics. Mol Cell Proteomics 2014;13:397-406.

5. Tanaka M, Murakami K, Ozaki S, et al. DIP2 discointeracting protein 2 homolog A (Drosophila) is a candidate receptor for follistatin-related protein/follistatinlike 1--analysis of their binding with TGF- $\beta$ superfamily proteins. FEBS J 2010;277:4278-89.

6. Liu Y, Tan X, Liu W, et al. Follistatin-like protein 1 plays a tumor suppressor role in clear-cell renal cell carcinoma. Chin J Cancer 2018;37:2.

7. Ni X, Cao X, Wu Y, Wu J. FSTL1 suppresses tumor cell proliferation, invasion and survival in non-small cell lung cancer. Oncol Rep 2018;39:13-20.

8. An J, Wang L, Zhao Y, et al. Effects of FSTL1 on cell proliferation in breast cancer cell line MDA-MB-231 and its brain metastatic variant MDA-MB-231-BR. Oncol Rep 2017;38:3001-10.

9. Jin $\mathrm{X}, \mathrm{Nie} \mathrm{E}, \mathrm{Zhou} \mathrm{X}$, et al. Fstl1 Promotes Glioma Growth Through the BMP4/Smad1/5/8 Signaling Pathway. Cell Physiol Biochem 2017;44:1616-28.

10. Chen SX, Xu XE, Wang XQ, et al. Identification of colonic fibroblast secretomes reveals secretory factors regulating colon cancer cell proliferation. J Proteomics 2014;110:155-71.

11. Torres S, Bartolomé RA, Mendes M, et al. Proteome Profiling of Cancer-Associated Fibroblasts Identifies Novel Proinflammatory Signatures and Prognostic Markers for Colorectal Cancer. Clin Cancer Res 2013;19:6006-19.

12. Kudo-Saito C. FSTL1 promotes bone metastasis by causing immune dysfunction. Oncoimmunology 2013;2:e26528.

13. Hao Y, Baker D, Ten Dijke P. TGF- $\beta$-Mediated EpithelialMesenchymal Transition and Cancer Metastasis. Int J Mol
Sci 2019. doi: 10.3390/ijms20112767.

14. Voutsadakis IA. The ubiquitin-proteasome system and signal transduction pathways regulating Epithelial Mesenchymal transition of cancer. J Biomed Sci 2012;19:67.

15. Zhao Y, Ou Q, Deng Y, et al. Determination of follistatinlike protein 1 expression in colorectal cancer and its association with clinical outcomes. Ann Transl Med 2019;7:606.

16. Voutsadakis IA. Pathogenesis of colorectal carcinoma and therapeutic implications: The roles of the ubiquitin-proteasome system and Cox-2.J Cell Mol Med 2007;11:252-85.

17. Beck SE, Jung BH, Del Rosario E, et al. BMP-induced growth suppression in colon cancer cells is mediated by p21WAF1 stabilization and modulated by RAS/ERK. Cell Signal 2007;19:1465-72.

18. Itatani Y, Kawada K, Sakai Y. Transforming Growth Factor- $\beta$ signaling pathway in colorectal cancer and its tumor microenvironment. Int J Mol Sci 2019;20:5822.

19. Cario E, Podolsky DK. Differential alteration in intestinal epithelial cell expression of toll-like receptor 3 (TLR3) and TLR4 in inflammatory bowel disease. Infect Immun 2000;68:7010-7.

20. Makkar S, Riehl TE, Chen B, et al. Hyaluronic Acid Binding to TLR4 Promotes Proliferation and Blocks Apoptosis in Colon Cancer. Mol Cancer Ther 2019;18:2446-56.

21. Koliaraki V, Chalkidi N, Henriques A, et al. Innate Sensing through Mesenchymal TLR4/MyD88 Signals Promotes Spontaneous Intestinal Tumorigenesis. Cell Rep 2019;26:536-45.e4.

22. Meyer C, Cagnon L, Costa-Nunes CM, et al. Frequencies of circulating MDSC correlate with clinical outcome of melanoma patients treated with ipilimumab. Cancer Immunol Immunother 2014;63:247-57.

23. Kudo-Saito C, Ishida A, Shouya Y, et al. Blocking the FSTL1-DIP2A Axis Improves Anti-tumor Immunity. Cell Rep 2018;24:1790-801.

24. Nie E, Miao F, Jin X, et al. Fstl1/DIP2A/MGMT signaling pathway plays important roles in temozolomide resistance in glioblastoma. Oncogene 2019;38:2706-21.

25. Larsson C, Akhtar Ali M, Pandzic T, et al. Loss of DIP2C in RKO cells stimulates changes in DNA methylation and epithelial mesenchymal transition. BMC Cancer 2017;17:487.

Cite this article as: Voutsadakis IA. A role for Follistatinlike protein 1 (FSTL1) in colorectal cancer. Ann Transl Med 2020;8(5):155. doi: 10.21037/atm.2020.01.112 